\title{
The Redo Antireflux Surgery after Failed Fundoplication
}

\section{Ion C. PUIA ${ }^{1,2}$, Aida PUIA ${ }^{2 *}$, Sebastian GHEORGHIU³ ${ }^{3}$ Paul G. CRISTEA ${ }^{1}$}

\author{
I"Octavian Fodor" Regional Institute of Gastroenterology and Hepatology, 3rd General Surgery Clinic, 19-21 Croitorilor Street, Cluj-Napoca, \\ Romania;drpuia@yahoo.fr;paulgcristea@gmail.com \\ 2 "Iuliu Hatieganu" University of Medicine and Pharmacy, 8 Victor Babes Street, Cluj-Napoca, \\ Romania;draidapuia@gmail.com ("corresponding author) \\ 3"Iuliu Hatieganu" University of Medicine and Pharmacy, 8 Victor Babes Street, Cluj-Napoca, Romania; seb@gheorghiu.ca
}

\begin{abstract}
The redo antireflux surgery is an advanced surgical procedure which enclosed the following conventional steps: the takedown of the previous fundoplication, the repair of the hiatal defect, followed by the actual antireflux procedure. In reoperations, a great percentage of surgeons favour the short floppy Nissen, although partial fundoplications or gastrojejunostomies are also practiced. A shortened esophagus is usually one of several causes for the reflux reoccurrence, and requires either an extended periesophageal dissection, or a Collis gastroplasty. The surface of the hiatal defect, rather than its diameter, is the most important key for its reconstruction. A large number of repair variants have been introduced and tested, showing that the antireflux surgery is still awaiting an ideal prosthetic material, whereas details regarding tailoring, placement and suturing of current meshes are subjects of debate. The most frequent complication in the redo antireflux surgery is the gastric perforation, but it is also possible that pulmonary or cardiac failure, infections, or acute pancreatitis, may appear. Mortality is around $1 \%$ for this procedure with a success rate of $80 \%$ and the surgery has a mean duration of $3-4$ hours even for experienced teams. Thus, due to its complexity and challenges, the redo antireflux surgery should be performed by an experienced team in a tertiary center to increase the favourable outcome.
\end{abstract}

Keywords: esophagus, fundoplication, gastroplasty, redo, reoperation

\section{Introduction}

The antireflux surgery for medical treatment of refractory gastroesophageal reflux disease (GERD) has a satisfactory outcome in up to $90 \%$ of patients. At least $10 \%$ will however experience recurrent reflux symptoms or dysphagia (Ferguson, 1997; Furnée et al., 2009; Patti et al., 2015). When medical treatment fails to relieve these symptoms, reoperation is recommended with reported rates varying between 6.9-15\% (Zhou et al., 2015; Richter, 2013).

An analysis of 3,175 reoperations (Furnée et al., 2009) mentioned the following causes for redo-surgery after a failed fundoplication: recurrent reflux (41.7\%), dysphagia (16.6\%), recurrent reflux and dysphagia (4.0\%), anatomical abnormalities $(2.5 \%)$ and gas bloat syndrome $(0.7 \%)$. The most frequent anatomical abnormalities representing the underlying causes of failure are: intrathoracic wrap migration $(27.9 \%)$, wrap disruption (22.7\%), telescoping (14.1\%), para-esophageal hiatal herniation $(6.1 \%)$, hiatal disruption $(5.3 \%)$, a tight wrap (5.3\%) and strictures (1.9\%) (Furnée et al., 2009).
The preoperative workup techniques are similar to those used in the patients undergoing evaluation for primary antireflux surgery.

Examining the patient at Ba-swallow together with an experienced radiologist's interpretation offers important information useful in selecting the appropriate surgical management. Different positions on the radiology table and selective pressure exerted on the abdomen are mandatory for a correct examination (Frantzides et al., 2006).

According to data offered by upper gastrointestinal (GI) series, failed fundoplication may be classified into six types: tight Nissen, incompetent repair, disruption of the wrap, stomach slippage above the diaphragm, slipped Nissen, and transdiaphragmatic wrap herniation (Carbo et al., 2014). Endoscopic application of radiopaque metal clips to mark the Z-line allows a precise visualization of the gastroesophageal junction area at the video contrast investigation (Barkhatov et al., 2015).

Esophagogastroduodenoscopy should usually follow Ba-swallow, while manometry is mandatory in patients with dysphagia and abnormal findings at the upper GI 
388

series. In addition, 24-hours $\mathrm{pH}$ monitoring is useful in diagnosing alkaline reflux (Barkhatov et al., 2015).

The aim of the hereby extensive review of current relevant literature was to discuss the indications, techniques, complications and limitations of this surgery. After over twenty years of experience in the laparoscopic approach of GERD, reoperations for failed fundoplications still represent a challenge. Based on the authors' expertise in open and laparoscopic redo operations, the most valuable ideas regarding surgical technique and procedure tactic were selected.

\section{The procedure steps of redo antireflux surgery}

The essential steps of redo antireflux surgery are: (1) removal of the previous fundoplication, (2) repair of the hiatal defect as needed, and (3) antireflux procedure (Mittal et al., 2013).

Most redo operations for failed antireflux procedures are performed by laparoscopy in tertiary specialized centers.

Considering the fact that postoperative adhesions may be present even in areas remote to the trocar entry points of the previous operation, blind penetration of the abdominal wall with the Veress needle or the first trocar may injure an adherent viscus. The insertion position of the next trocars must be chosen carefully, considering the conditions of the initial fundoplication. A forceful retraction of the liver may tear adhesions on the inferior surface. The spleen is rarely at risk during adhesiolysis (Furnée et al., 2009) but traction on the larger curvature should be made only after transecting adhesions on the spleen.

The hiatal dissection is usually started on the right crus and progression has to follow the correct plane keeping the perimysium on the muscle fibers. Whereas difficulties in delineating the esogastric junction been encountered, a lighted bougie may facilitate the identification of the esophagus. The introduction of this bougie should be done by endoscopy in order to avoid tearing the inferior oesophagus (Frantzides et al., 2006).

Once the fundoplication is identified, anatomy can be restored by removing the stitches securing the wrap. The ultrasonic scissors are the most appropriate tool for this procedure as the thermal damage produced is minimal. If present, the short gastric vessels should be divided to allow the creation of a tension free plication. Together with the identification of the angle of Hiss, this procedure is essential for a correct fundus-to-fundus wrap (Frantzides et al., 2006).

The type 1 esophageal dissection is limited to the last 4 $\mathrm{cm}$ of the organ if the necessary 2.5 centimetres of abdominal esophagus are obtained. In case of a short esophagus (SE) a type 3 transhiatal dissection may reach even the carina. If this action it's not enough as a lengthening procedure, a Collis gastroplasty will be instead performed (Puri et al., 2013).

The hiatal defect is sutured and a mesh may be used in order to avoid hiatal disruption. The self-locking sutures might be helpful avoiding relaxation of the knots if tension is present. The figure-of-eight sutures are recommended by some authors (Juhasz et al., 2012) however, they may cause ischemia of the muscle fibers.

The calibration of the mesh must be loose enough to allow a $10 \mathrm{~mm}$ forceps to pass between the esophagus and the crura. Another variant consists in using an intralumenal 60-Fr bougie instead (Mittal et al., 2013).

A prospective double-blind randomized control trial concluded that the anterior suturing of the hiatus is comparable in the short-term to that of the posterior type (Watson et al., 2001). However, an interruption of the muscle fiber loop by the placement of anterior sutures seems to render the hiatal musculature less functional.

The fundoplication is performed using two or three stitches and most authors incorporate the esophagus with one of them. Some authors consider that this procedure might increase the risk for subsequent dysphagia (Frantzides et al., 2006).

The gastropexy consisting in suturing the wrap to the right crus in order to avoid slippage should also be avoided as it is considered that these partner organs have independent motility (Frantzides et al., 2006).

\section{The challenges of the antireflux mechanism}

The 360-type short floppy Nissen fundoplication seems to be favoured in over $50 \%$ of reoperations. Partial fundoplication (Toupet, Dor - 21.8\%) (Furnée et al., 2009) is used when the patient is also diagnosed with an impaired esophageal motility (Livingston et al., 2001). Several studies have demonstrated that total fundoplication is associated with a slightly better reflux control than partial wraps but is associated with a higher percentage of dysphagia (Shukri et al., 2008).

The resection surgery is quite rare (7.1\%) and has limited indications. Some examples include cases of severe esophagitis, Barrett's esophagus, peptic stricture of the esophagus, severely disturbed esophageal motility or a short esophagus. In a study of 202 reoperations, gastric resection was recommended to patients with alkaline reflux, dense adhesions on previously attempted refundoplications or those with severe gastric paresis (Furnée et al., 2009).

In the case of delayed gastric emptying, a history of gagging or retching, a short esophagus especially with decreased motility, morbid obesity, a failed Collis gastroplasty, or a history of three or more failed previous antireflux surgeries, a Roux-en-Y loop (RNY) is indicated instead of fundoplication (Mittal et al., 2013). The preferred method is a gastrojejunostomy (GJ) with a longitudinal pouch of $60-75 \mathrm{~cm}^{3}$. A redo-fundoplication (RF) on the proximal gastric stump may be performed; however, this is not necessary since the RNY is sufficient as an antireflux procedure. When the hyper acidity represents a concern, a near-esophagojejunostomy (EJ) with an extremely small gastric pouch of $5-10 \mathrm{ml}$ capacity may serve as a better alternative (Makris et al., 2009). The RNY esophagojejunostomy is also an alternative procedure in cases of esophageal strictures or irreparable damage to the cardia as a distal esophagectomy and a proximal gastrectomy are also necessary to be performed in these cases (Mittal et al., 2013). 
The standard RNY lengths are $20 \mathrm{~cm}$ for the biliary limb and $60 \mathrm{~cm}$ for the alimentary limb. A longer alimentary limb is usually recommended in obese patients. In open surgery, retrocolic placement is the standard method whereas antecolic placement is easier to perform in laparoscopical surgery (Mittal et al., 2013). Closure of mesenteric defects is mandatory in order to avoid internal herniation.

A temporary gastrostomy is useful in maintaining the nutritional status of the patient along with providing a convenient route for the drug administration. An advantage of sparing the stomach is that a gastric tube remains available for use in esophagoplasty for those patients where a future esophagectomy might be necessary. On the other hand, an isolated distal stomach proves impossible to explore by endoscopic means (Shukri et al., 2008; Mittal et al., 2013).

The tertiary centers with expertise in redo-surgeries after failed fundoplications have demonstrated that RNY offers similar results to RF (Mittal et al., 2013; Grover and Kothari, 2015).

\section{The challenges of the shortened oesophagus}

The role of the shortened esophagus (SE) in the reoccurrence of GERD by mechanism of herniation, the plication slippage or the hiatal disruption, has been heavily debated over the past 50 years.

Esophageal shortening is triggered by inflammation secondary to acid reflux. Initially limited to the mucosa, it can gradually affect all layers of the esophagus. The variable degree of fibrosis may lead to retraction in a circular or longitudinal manner, thus causing either stenosis or shortening (Horvath et al., 2000).

The frequency of esophageal shortening remains unclear as a very wide range of values from $0 \%$ to $60 \%$ are reported. Several authors described and introduced lengthening procedures in $57 \%$ of the cases (Nason et al., 2011) whereas others deny the existence of the nonreducible esophagus (Madan et al., 2004; Frantzides et al., 2006).

Most of the studies originating in tertiary specialized centres describe a more balanced attitude, with only $10 \%$ of patients requiring transhiatal dissection and 5\% requiring gastroplasty (Johnson et al., 2000; Awad et al., 2001). A tendency may result from the fact that these units concentrate more difficult cases which are referred from the surrounding, less specialized hospitals. On the other hand the availability of new generation laparoscopic suturing devices may favour the use of gastroplasty techniques, thus raising the percentage of lengthening procedures currently performed.

The preoperative examinations of patients with a suspicion of a short esophagus are similar to those for GERD (Kunio et al., 2015). Preoperative indicators of a short esophagus are: a history of previously failed antireflux surgery, a gastroesophageal junction more than $5 \mathrm{~cm}$ above the diaphragmatic hiatus, a large type I or type III hiatal hernia, severe esophagitis, a distal esophagus with hypoperistalsis or aperistalsis, peptic stricture or metaplastic changes (Horvath et al., 2000).
Even in the presence of all these factors, a preoperative diagnosis of a true short esophagus cannot be assured, and an objective diagnosis of a SE is made intraoperatively following adequate mediastinal mobilization; therefore, the surgical team must be prepared for the necessity of a lengthening procedure.

A short esophagus is defined on Ba-swallow by a gastro esophageal junction (GEJ) located superior to the diaphragmatic hiatus. It can be classified into three types: an apparent short esophagus, a true but reducible short esophagus and a true, nonreducible short esophagus (Horvath et al., 2000). For the first type, a standard esophageal dissection of the distal $4 \mathrm{~cm}$ is enough in achieving the minimum $2.5 \mathrm{~cm}$ length needed for the intraabdominal esophagus. For the second type a periesophageal dissection, ascending to the level of the carina is necessary in order to obtain the minimum length. In case of a true, nonreducible short esophagus, even a high reaching, intramediastinal dissection proves unable to achieve the desired minimum length, thus a gastroplasty becomes mandatory (Kohn et al., 2013).

The surgical treatment of a SE is oriented towards lengthening procedures consisting of either an extended periesophageal dissection or a Collis procedure. The esophagectomy is seldom applied. Intrathoracic fundoplication as an alternative procedure should be avoided due to a high rate of complications (strangulation, perforation, ulceration, bleeding) as well as a precarious quality of life for the patient (epigastric or chest pain, dysphagia) (Horvath et al., 2000). An interesting lengthening procedure consisting of a complete circular myotomy just below the level of the aortic arch, in combination with a Belsey Mark IV antireflux repair has yielded promising results in a small scale study (Allen and Matthews, 1993).

Prior to the advent of videolaparoscopy, high periesophageal dissection was performed by thoracotomy with the esophagus being mobilized to the level of the aortic arch. This procedure was favoured since a safely performed transhiatal dissection could not reach high enough. Currently the dissection is performed via thoracoscopy as a preservation of a tradition linked to the thoracic approach (Juhasz et al., 2010). However, most surgeons nowadays favour the laparoscopic transhiatal access instead. Still, the presence of massive morbid obesity, multiple prior foregut operations, extremely foreshortened esophagus, and massive herniation of multiple abdominal viscera generally indicate a thoracotomy as the initial approach (Puri et al., 2013). The use of a plastic sling passed under the GEJ is quite useful in exposing the area during dissection but bears the risk of excessive traction (Bjelović et al., 2014). This would lead to an apparent gain in length by elongating the esophagus as a result of its elasticity. A simple check consists in releasing any tension on the organ before measuring the intra-abdominal segment. Misidentification of the proximal stomach with the esophagus is caused by the insufficient resection of the hernial sac and fat pad (Whitson et al., 2006) and may favour an incorrect placement of the fundoplication around the upper gastric pole. 
The Collis gastroplasty is considered an alternative procedure if a high periesophageal dissection fails to provide the required minimum $2.5 \mathrm{~cm}$ of intraabdominal esophagus. Pearson was the first who combined the Belsey plication with a Collis procedure (Pearson et al., 1971) and later reported excellent results with this method.

In 1996, Swanstrom and his team (Swanstrom et al., 1996) were the first who described a combined laparoscopic and thoracoscopic approach for a CollisNissen procedure. After the transection of the short gastric vessels, the fundus must be completely mobilized in order to allow transhiatal access of the stapling device on the stomach which is rotated in an anterior direction. The first thoracic trocar must be placed following the usual precautions required when pleural adhesions may be present. A selective intubation is usually not necessary as a low insufflation pressure collapses the right lung. An articulating stapler provides the surgeon more freedom in choosing the thoracic entry point for the $12 \mathrm{~mm}$ trocar. Otherwise, in order to obtain an appropriate angle, the trocar must be placed high on the midaxillary line. Two operative telescopes allow a safer penetration through the mediastinal pleura. A calibrating bougie of 48-54 Fr pushed close to the lesser curvature is mandatory. A 30 $\mathrm{mm}$ blue cartridge should be used as the gastric wall is thin at this level. The suture line must be checked for haemorrhage on both sides. The distention caused by the bougie may impair the bleeding so the line on the neoesophagus should be checked again after removing the bougie. Oversewing the suture line is not necessary. During the completion of a Nissen fundoplication, the excessive traction on the suture line should be avoided.

Johnson and Hunter (Johnson et al., 1996) have developed a totally laparoscopic approach resembling a vertically banded gastroplasty. After a standard preparation of the fundus, a circular stapler is fired at an appropriate distance to the lesser curvature and the Hiss angle. The breach obtained allows the passage of an Endo-GIA stapling device which will be positioned parallel to the lesser curvature, ascending to the Hiss angle.

A Collis variant in which the neoesophagus is obtained by performing a wedge resection of the fundus uses two or three firings of the articulated Endo-GIA. The first starts from the larger curvature, the second continues towards the lesser curvature and the last reaches the Hiss angle (Whitson et al., 2006; Puri et al., 2013).

The uncut Collis-Nissen gastroplasty has an acceptable morbidity and excellent short and long-term results (Trastek, 1995).

The long-term complications encountered after a Collis gastroplasty include: the distal esophageal aperistalsis with dilatation and dysphagia. The persistence of acid-producing gastric mucosa above the wrap may cause heartburn and asymptomatic recurrence (Jobe et al., 1998; Puri et al., 2013).

\section{The challenges of the hiatal defect}

Even before the first published studies regarding the complications of esophageal erosions due to prosthetic materials were conducted, the presence of mesh used in hiatoplasties raised worries. Large unicentric studies reported few or even no mesh-related complications (Granderath et al., 2003; Juhasz et al., 2010). However, observations suggest that complications from synthetic mesh placed in this location may be more common than previously thought. Regardless the tailoring of the mesh, complications sometimes simply arise. The heart-shaped, the keyhole or the horseshoe meshes have all been described in cases in which erosions, stenosis or dense fibrosis have occurred.

In cases where crural approximation is difficult, mesh may be used as a bridge or as an interposition (Kohn et al., 2013) however, both methods still produce the above mentioned complications (Stadlhuber et al., 2009).

Non-absorbable sutures used to secure the mesh are safer than staples which are prone to causing complications by penetrating the diaphragmatic vessels. Teflon pledgets may be used in order to avoid sutures cutting through the muscle fibers; esophageal erosions tend to occur as a complication, however without clinical consequences (Juhasz et al., 2010).

Although the distance of the mesh edges from the esophagus is potentially an important aspect to consider, most of the available studies fail to mention or provide precise data regarding this. A possible mechanism in which the edge of the mesh slowly cuts the esophagus if applied too close it has been described. The shrinkage, the migration or strong diaphragmatic contractions may produce the same effect even in correctly applied meshes (Stadlhuber et al., 2009).

All the types of mesh, whether synthetic (polypropylene, polytetrafluoroethylene, dual) or biological may lead to complications (Stadlhuber et al., 2009). The sterilized human dermal collagen allows spontaneous closure of small fistulae, provokes minimal fibrosis thus strictures, dysphagia or adhesions are seldom observed (Ringley et al., 2006; Bell et al., 2013).

The most frequent symptoms associated with mesh complications are dysphagia, heartburn, and chest pain. Fever, epigastric pain and weight loss can also be encountered (Stadlhuber et al., 2009).

Regarding the surgical tactic, if mesh complications require a distal esophagectomy, RNY esojejunostomy seems to be the favoured surgical approach. If the upper gastric pole is resected as well, the distal stomach may be left in situ (Juhasz et al., 2010).

Although the routine mesh use is encouraged by some authors (Johnson et al., 2006), a more balanced attitude recommends bioprosthesis usage in the hiatal disruptions, large paraesophageal hernias or tearing at crus closure (Frantzides et al., 2006; Juhasz et al., 2010).

The guidelines for the minimal dimension of the hiatus requiring a mesh repair should consider the hiatal surface more than its maximal diameter (Kohn et al., 2013). An autologous graft with fascia lata may offer the ideal solution. The available data is promising, but results on a larger scale have yet to confirm these initial good results (Bjelović et al., 2014).

Although the details regarding tailoring, placement and suturing of the current available materials remain 
largely debated, ideal prosthetic material used in antireflux surgery has yet to be discovered.

The conversion rate from laparoscopic to open surgery is low for experienced teams (1.4-8.7\%) and is mostly attributed to dense adhesions, severe intraoperative bleeding or poor visualization of the surgical field (Frantzides et al., 2006; Furnée et al., 2009).

The gastric perforation is the most frequent intraoperative complication and can be treated through laparoscopic surgery, although the use of mesh is contraindicated due to possible bacterial contamination (Frantzides et al., 2006).

The redo-antireflux surgery has a mean duration of 3-4 hours even for experienced teams (Furnée et al., 2009; Juhasz et al., 2010) with postoperative mortality rates approximately around 1\% (Furnée et al., 2009).

Infectious, pulmonary, cardiac complications or acute pancreatitis can affect up to $15 \%$ of the patients (Hughes et al., 1999). Symptomatic outcome after reoperation is reported as successful in up to $80 \%$ of patients (Awad et al., 2001; Furnée et al., 2009; Dallemagne et al., 2011).

\section{Conclusions}

The redo-antireflux surgery has a higher morbidity and mortality rate than primary antireflux surgery. A correct preoperative assessment of the patient, along with the presence of adhesions, shortening of the esophagus and an enlarged hiatus are the main issues which must be solved. An increased effort in collecting prospective data could lead to better technical and tactical solutions.

\section{Acknowledgements}

This research received no specific grant from any funding agency in the public, commercial, or not-forprofit sectors.

\section{References}

Allen SM, Matthews HR (1993). Circular myotomy and Belsey repair for acquired shortening of the oesophagus. European Journal of Cardio-Thoracic Surgery 7(12):645-647.

Awad ZT, Mittal SK, Roth TA, Anderson PI, Wilfley WA, Filipi CJ (2001). Esophageal shortening during the era of laparoscopic surgery. World Journal of Surgery 25(5):558-561.

Barkhatov L, Kazaryan AM, Aasen S, Edwin B (2015). Endoscopic clipping of the Z-line (CMZL) helps recognize anatomical failures after Nissen fundoplication: technical report of a new method. Wideochirurgia $\mathrm{i}$ inne techniki małoinwazyjne 10(3):363-367.

Bell RC, Fearon J, Freeman KD (2013). Allograft dermal matrix hiatoplasty during laparoscopic primary fundoplication, paraesophageal hernia repair, and reoperation for failed hiatal hernia repair. Surgical Endoscopy 27(6):1997-2004.

Bjelović M, Babic T, Gunjić D, Veselinović M, Spica B (2014). Laparoscopic repair of hiatal hernias: experience after 200 consecutive cases. Srpski arhiv za celokupno lekarstvo 142(78):424-430.
Carbo AI, Kim RH, Gates T, D'agostino HR (2014). Imaging findings of successful and failed fundoplication. Radiographics 34(7):1873-1884.

Dallemagne B, Arenas Sanchez M, Francart D, Perretta S, Weerts J, Markiewicz S, Jehaes C (2011). Long-term results after laparoscopic reoperation for failed antireflux procedures. British Journal of Surgery 98(11):1581-1587.

Ferguson MK (1997). Pitfalls and complications of antireflux surgery. Nissen and Collis-Nissen techniques. Chest Surgery Clinics of North America 7(3):489-509.

Frantzides CT, Carlson MA, Zografakis JG, Moore RE, Zeni T, Madan AK (2006). Postoperative gastrointestinal complaints after laparoscopic Nissen fundoplication. Journal of the Society of Laparoendoscopic Surgeons 10(1):39-42.

Furnée EJ, Draaisma WA, Broeders IA, Gooszen HG (2009). Surgical reintervention after failed antireflux surgery: a systematic review of the literature. Journal of Gastrointestinal Surgery 13(8):1539-1549.

Granderath FA, Kamolz T, Schweiger UM, Pointner R (2003). Laparoscopic refundoplication with prosthetic hiatal closure for recurrent hiatal hernia after primary failed antireflux surgery. Archives of Surgery 138(8):902-907.

Grover BT, Kothari SN (2015). Reoperative antireflux surgery. Surgical Clinics of North America 95(3):629-640.

Horvath KD, Swanstrom LL, Jobe BA (2000). The short esophagus: pathophysiology, incidence, presentation, and treatment in the era of laparoscopic antireflux surgery. Annals of Surgery 232(5):630-640.

Hughes SG, Chekan EG, Ali A, Reintgen KL, Eubanks WS (1999). Unusual complications following laparoscopic Nissen fundoplication. Surgical Laparoscopy Endoscopy \& Percutaneous Techniques 9(2):143-147.

Jobe BA, Horvath KD, Swanstrom LL (1998). Postoperative function following laparoscopic collis gastroplasty for shortened esophagus. Archives of Surgery 133(8):867-874.

Johnson AB, Oddsdottir M, Hunter JG (1998). Laparoscopic Collis gastroplasty and Nissen fundoplication. A new technique for the management of esophageal foreshortening. Surgical Endoscopy 12(8):1055-1060.

Johnson JM, Carbonell AM, Carmody BJ, Jamal MK, Maher JW, Kellum JM, DeMaria EJ (2006). Laparoscopic mesh hiatoplasty for paraesophageal hernias and fundoplications: a critical analysis of the available literature. Surgical Endoscopy 20(3):362-366.

Juhasz A, Sundaram A, Hoshino M, Lee TH, Mittal SK (2012). Outcomes of surgical management of symptomatic large recurrent hiatus hernia. Surgical Endoscopy 26(6):1501-1508.

Kohn GP, Price RR, DeMeester SR, Zehetner J, Muensterer OJ, Awad Z, Mittal SK, Richardson WS, Stefanidis D, Fanelli RD; SAGES Guidelines Committee (2013). Guidelines for the management of hiatal hernia. Surgical Endoscopy 27(12):44094428.

Kunio NR, Dolan JP, Hunter JG (2015). Short esophagus. Surgical Clinics of North America 95(3):641-652. 
Livingston CD, Jones HL, Askew RE, Victor BE, Askew RE (2001). Laparoscopic hiatal hernia repair in patients with poor esophageal motility or paraesophageal herniation. The American Journal of Surgery 67(10):987-991.

Madan AK, Frantzides CT, Patsavas KL (2004). The myth of the short esophagus. Surgical Endoscopy 18(1):31-34.

Makris KI, Lee T, Mittal SK (2009). Roux-en-Y reconstruction for failed fundoplication. Journal of Gastrointestinal Surgery 13(12):2226-2232.

Mittal SK, Légner A, Tsuboi K, Juhasz A, Bathla L, Lee TH (2013). Roux-en-Y reconstruction is superior to redo fundoplication in a subset of patients with failed antireflux surgery. Surgical Endoscopy 27(3):927-935.

Nason KS, Luketich JD, Awais O, Abbas G, Pennathur A, Landreneau RJ, Schuchert MJ (2011). Quality of life after Collis gastroplasty for short esophagus in patients with paraesophageal hernia. The Annals of Thoracic Surgery 92(5):1854-1860.

Patti MG, Allaix ME, Fisichella PM (2015). Analysis of the causes of failed antireflux surgery and the principles of treatment: A Review. Journal of the American Medical Association Surgery 150(6):585-590.

Pearson FG, Langer B, Henderson RD (1971). Gastroplasty and Belsey hiatus hernia repair. An operation for the management of peptic stricture with acquired short esophagus. The Journal of Thoracic and Cardiovascular Surgery 61(1):50.

Puri V, Jacobsen K, Bell JM, Crabtree TD, Kreisel D, Krupnick AS, Patterson GA, Meyers BF (2013). Hiatal hernia repair with or without esophageal lengthening: is there a difference?. Innovations : technology and techniques in cardiothoracic and vascular surgery $8(5): 341-347$.

Richter JE (2013). Gastroesophageal reflux disease treatment: side effects and complications of fundoplication. Clinical Gastroenterology and Hepatology 11(5):465-471.
Ringley CD, Bochkarev V, Ahmed SI, Vitamvas ML, Oleynikov D (2006). Laparoscopic hiatal hernia repair with human acellular dermal matrix patch: our initial experience. American Journal of Surgery 192(6):767-772.

Shukri MJ, Watson DI, Lally CJ, Devitt PG, Jamieson GG (2008). Laparoscopic anterior 90 degree fundoplication for reflux or large hiatus hernia. Australian and New Zealand Journal of Surgery 78(3):123-127.

Stadlhuber RJ, Sherif AE, Mittal SK, Fitzgibbons RJ Jr, Michael Brunt L, Hunter JG, Demeester TR, Swanstrom LL, Daniel Smith C, Filipi CJ (2009). Mesh complications after prosthetic reinforcement of hiatal closure: a 28-case series. Surgical Endoscopy 23(6):1219-1226.

Swanstrom LL, Marcus DR, Galloway GQ (1996). Laparoscopic Collis gastroplasty is the treatment of choice for the shortened esophagus. American Journal of Surgery 171(5):477-481.

Trastek VF (1995). Uncut Collis-Nissen gastroplasty. Chest surgery clinics of North America 5(3):423-435.

Watson DI, Jamieson GG, Devitt PG, Kennedy JA, Ellis T, Ackroyd R, Lafullarde T, Game PA (2001). A prospective randomized trial of laparoscopic Nissen fundoplication with anterior vs posterior hiatal repair. Archives of Surgery 136(7):745-751.

Whitson BA, Hoang CD, Boettcher AK, Dahlberg PS, Andrade RS, Maddaus MA (2006). Wedge gastroplasty and reinforced crural repair: important components of laparoscopic giant or recurrent hiatal hernia repair. The Journal of Thoracic and Cardiovascular Surgery 132(5):1196-1202.

Zhou T, Harnsberger C, Broderick R, Fuchs H, Talamini M, Jacobsen G, Horgan S, Chang D, Sandler B (2015). Reoperation rates after laparoscopic fundoplication. Surgical Endoscopy 29(3):510-514. 